\title{
Influence of the "Slingshot" bench press training aid on bench press kinematics and neuromuscular activity in competitive powerlifters.
}

Running head: Impact of the Slingshot on bench press performance

James H. Dugdale, Angus Hunter, Thomas Di Virgilio, Lewis J. Macgregor and D. Lee Hamilton*

Physiology, Exercise \& Nutrition Research Group, Faculty of Health Sciences and Sport, University of Stirling, Stirling, UK, FK9 4LA.

* To whom correspondence should be addressed

D. Lee Hamilton

Room 3A60,

The Cottrell Building,

University of Stirling,

Stirling,

UK,

FK9 4LA.

Tel: 01786466475

Email: d.l.hamilton@stir.ac.uk

Publisher policy allows this work to be made available in this repository. This is not the final published version. Dugdale JH, Hunter A, Di Virgilio T, Macgregor LJ \& Hamilton DL (2019) Influence of the "Slingshot" bench press training aid on bench press kinematics and neuromuscular activity in competitive powerlifters. Journal of Strength and Conditioning Research, 33 (2), pp. 327-336. The final published version can be found at: https://doi.org/10.1519/JSC.0000000000001853 


\section{Abstract:}

This study examined the acute effects of the 'Slingshot' on bench-press performance, prime-mover surface electromyographic (sEMG) amplitude, and barbell velocity during maximal and submaximal bench-pressing in competitive male powerlifters. Fifteen male powerlifters (mean \pm SD age: $27.05 \pm 5.94$ years; mass: $94.15 \mathrm{~kg}$; $1 \mathrm{RM}$ bench-press: $139.7 \pm 16.79 \mathrm{~kg}$ ) participated in the study. Bench-press strength, average barbell velocity, and sEMG amplitude of the prime mover muscles (triceps brachii, pectoralis major and anterior deltoid) were measured during two conditions; 'Raw' (without use of any assistance) and 'Slingshot' [using the 'Slingshot' to perform both the weight achieved during 'Raw' 1RM testing (Raw max/SS), and absolute 1RM using the 'Slingshot' (SS)]. The results showed that the 'Slingshot' significantly increased bench press 1RM performance by a mean \pm SD of $20.67 \mathrm{~kg} \pm 3.4 \mathrm{~kg}$. Barbell velocity and stick point analysis indicate that this improvement is likely driven by an increase in peak and pre-stick barbell velocity as triceps RMS was lower throughout all rep max phases with the 'Slingshot'. The 'Slingshot' also caused reductions in RMS, specifically of the triceps at all rep ranges but barbell velocity was better maintained in the last reps of all sets. These data indicate that the 'Slingshot' specifically de-loaded the triceps muscle throughout all rep ranges and provide assistance to maintaining barbell velocity under fatigue during later repetitions of multiple-repetition sets. The 'Slingshot' training aid could therefore be used in de-load phases of bench press training or as an over-reaching and velocity training aid.

Key words: Stick point, stick period, powerlifting, bench-press, slingshot 
2 The bench press is one of the most utilised exercises within strength and

3 conditioning practice and programming (5). Similar to other free-weight

4 resistance exercises, the bench press is utilised for developing maximal strength,

5 power, and hypertrophy (30). The bench press is also one of the three

6 competition lifts within the sport of powerlifting (IPF, 2015). However, the

7 popularity of the bench press is due to its ability to develop the strength, power,

8 and hypertrophy of the prime movers: the pectoralis major, anterior deltoid, and

9 triceps brachii $(14,21,23,25)$. Several studies demonstrate the transfer of bench

10 press strength to improvements in motor unit recruitment through various

11 planes of the shoulder $(14,15)$, and more importantly for athletic performance,

12 strength in the bench press is an indicator of performance in strength and power

13 sports $(11,12,22)$. Therefore developing strategies to improve bench press

14 performance has the potential to improve performance across a range of sports

15 including but not limited to powerlifting, discus throwing (11), swimming (12),

16 and kayaking (22).

17 When training for an increase in strength, several training methods and 18 strategies can be adopted. With regards to specificity and technical practice it is 19 important to perform the full movement itself, however there is a growing trend 20 to utilize supplementary or assistance training to develop the muscles, 21 movement patterns, or weak points within a given exercise $(28,29,34)$. A recent 22 survey of competitive powerlifters demonstrated that over $50 \%$ are utilizing 
resistance bands in their bench press training, more so than alternative supplementary training methods such as the use of chains (29).

Elastic resistance training primarily involves the use of elastic bands of varied thicknesses to challenge a movement pattern and align with the force capability of the musculature throughout the range of motion of many movement tasks (28, 34). Several studies demonstrate that the use of combined elastic resistance training in the bench press improves the development of upper body strength 1 $9,13,18)$, and in addition, a recent meta-analysis supports the efficacy of variable resistance training methods (use of bands and chains) to improve measures of maximal strength (27). Despite the increased popularity and evidence for the use of elastic resistance training, far less attention has been focused on elastic assistance training.

Elastic assistance training utilises an assistance or an over-speed approach during the performance of athletic and strength training movements, allowing an athlete to run faster, jump higher, or lift more weight than they could do without the assistance $(8,31)$. Several studies demonstrate that elastic assistance acutely improves jump height (31) and sprinting performance (8), whilst chronic jump training with elastic assistance for 4-weeks significantly improved jump performance compared to training without assistance (3). Relative to research on elastic resistance devices, much less attention has been given to the implementation of elastic assistance devices for upper-body strength performance.

A recent study examined the acute effects of implementing a supportive assistance device called the 'Slingshot', on 1RM bench press performance in 19 
resistance-trained male participants (35). The study observed the effect of the 'Slingshot' in comparison to traditional 'Raw' bench press performance, and report significant increases to $1 \mathrm{RM}$ and barbell velocity associated with trends for decreased EMG amplitude for both the pectoralis major and triceps brachii. They report that all participants showed an increase in absolute 1RM performance by an average of $\sim 16 \mathrm{Kg}$ whilst wearing the 'Slingshot', and that participants were able to execute their 'Raw' $1 \mathrm{RM}$ weight at significantly higher barbell velocity and power output when using the 'Slingshot'. However, when the relative intensity was matched between the absolute 'Raw' vs. 'Slingshot' 1RM average barbell velocity and average power output were not statistically different and there was a trend for the prime mover normalised EMG amplitude to be lower whilst wearing the 'Slingshot' despite the heavier load. These data indicate that the 'Slingshot' was assisting participants to lift either heavier loads or equal loads at a greater velocity, whilst the trends for decreased EMG amplitude suggest potential de-loading in the prime movers.

We therefore assessed bench press kinematics and neuromuscular activation during maximal and submaximal bench-pressing with or without the 'Slingshot' in trained powerlifters. Our aim was to use stick point analysis $(10,32)$ in conjunction with EMG assessments to try to understand the mechanism by which the 'Slingshot' improves 1RM, and the influence it may have on matched intensity submaximal sets. We hypothesized that the improvement in 1RM with the 'Slingshot' would be due to either A) an increased normalised sEMG amplitude of the prime movers during or after the stick-period of the benchpress or B) that the improvement would be due to a greater peak and average 
velocity in the early phases of the bench press as a result of the elastic assistance provided by the 'Slingshot'. As a secondary hypothesis we also theorized that the 'Slingshot' would maintain barbell velocity during sets with multiple repetitions.

\section{Methods:}

\section{Experimental Approach to the problem}

This study utilised a within-subjects design to examine the effects of Mark Bell's original 'Slingshot' on maximal and sub-maximal bench press kinematics and neuromuscular activity. The study was designed to assess how using the elastic assistance device, the 'Slingshot', altered neuromuscular recruitment patterns of the prime movers and the kinematics of the bench press during maximal and submaximal efforts. These measurements will allow us to determine the mechanism by which the 'Slingshot' may be working and illustrate what affect it may have on muscle recruitment.

\section{Subjects}

The methods and procedures implemented within this study were approved by the University of Stirling, School of Sport Research Ethics Committee and all participants provided informed consent upon recruitment selection. All testing took place at the Gannochy Sports Centre - Athlete Performance Laboratory, at the University of Stirling, Scotland, UK. Fifteen male competitive powerlifters (mean \pm SD: age $=27.05 \pm 5.94 \mathrm{yrs} ;$ body mass $=94.15 \pm 13.43 \mathrm{~kg} ;$ height $=$ $177.38 \pm 4.33 \mathrm{~cm} ; 1 \mathrm{RM}=139.7 \pm 4.34 \mathrm{~kg}$ ) voluntarily participated in this study. Participants were contacted through word of mouth, social media, and through 
study advertisement from 2 drug-tested powerlifting federations within the UK. Participants were selected based upon having $\geq 2$ years powerlifting-based strength training. All participants were considered healthy and injury-free based upon their responses to a Physical Activity Readiness Questionnaire (PAR-Q) and understood no reason as to why their ability to exert maximal bench press force would be limited in any way.

$$
\text { *** Table } 1 \text { about here }{ }^{* * *}
$$

\section{Procedures}

The study consisted of two laboratory based trials of $\sim 1.5$ hours each. Trials were scheduled between $7<14$ days apart and were completed at the same time of day to account for circadian variation (4). During each trial, participants' 1RM bench press was measured, followed by a predicted 3RM (3Rep) at $87.5 \%$ of achieved 1RM and 3 sub-maximal sets of 8 repetitions (8Rep) at 70\% of achieved 1RM (2). All participants completed the 'Raw' trial first (without the use of the Slingshot), followed by the 'Slingshot' (SS) trial.

All bench press attempts were completed on a solid leather competition height bench secured in position inside a FT700 Power Cage (Fitness Technology, Australia), and using an IPF specification Eleiko PL competition barbell (Eleiko, Sweden), Eleiko WL coloured training discs (Eleiko, Sweden), and Eleiko Olympic WL competition collars (Eleiko, Sweden).

Prior to commencing the initial trial, participants were provided with a 3-day training and food diary, and asked to complete both diaries in the 2 days leading up to, and day of testing. Participants were advised to maintain their normal diet 
and training habits and to avoid completing the bench press exercise 48 hours

118 prior to testing. Prior to the second trial participants were provided with their

119 original diaries and advised to replicate their activities to the upmost of their 120 ability.

121 Upon arrival to the laboratory, participants provided anthropometric measurements comprised of their age (years), body mass $(\mathrm{kg})$, and height $(\mathrm{cm})$, 123 along with a competition-style bench press $1 \mathrm{RM}(\mathrm{kg})$ predicted to the best of 124 their ability. Participants were also allowed to select their preferred rack height, 125 and demonstrated their bench press grip width, which was measured and recorded $(\mathrm{cm})$ and marked for reference on the barbell using masking tape.

127 Prior to commencing any warm-up activities, participants were familiarised with 128 the testing protocol and requirements, and allowed to ask any questions or for 129 any further information if required. During the initial phase of the warm-up, all 130 participants were required to familiarise themselves with the sEMG normalisation procedure by completing controlled and consistent bench press 132 repetitions using the empty barbell to a metronome set at $30 \mathrm{bpm}$, prior to 133 loading. During this familiarisation, a clearly audible metronome was played 134 through a pair of speakers, and participants were required to complete a full 135 competition style set up, un-rack the barbell, and perform as many repetitions as 136 they deemed necessary until they felt confident executing the bench press 137 movement to the rhythm of the metronome. Each time the metronome sounded 138 indicated a change of phase (eccentric: concentric), requiring a controlled time 139 period of 2 s per phase. 
Following the metronome familiarisation, the barbell was loaded for participants

141 and they performed repetitions at various increments of their choosing. Number

142 of sets, repetitions, and loadings were recorded on a data collection sheet for

143 replication in the subsequent trial and rest intervals of 2-minutes were provided

144 throughout the warm up. For normalisation purposes, participants were

145 recorded performing one set of five repetitions at $70 \%$ of predicted $1 \mathrm{RM}$ to the

$14630 \mathrm{bpm}$ metronome, following the criteria highlighted above (7). Following the

147 single normalisation set, participants continued with their own self-selected

148 warm up.

149 Once participants exceeded 90\% predicted 1RM, all attempts were considered

150 '1RM attempts' and were completed between 5-minute rest intervals and to

151 correct referee commands and competition rules (IPF, 2015). Participants

152 proceeded with $1 \mathrm{RM}$ attempts in increments of their choosing and in agreement

153 with the primary investigator until they reached muscular or technical failure. In

154 all instances, 1RM was achieved between 3-5 attempts. Attempts were

155 disqualified if the participant failed to successfully perform the repetition or if

156 they failed to meet all competition requirements for successful bench press

157 performance (IPF, 2015). Once participants had established a $1 \mathrm{RM}$, they

158 performed three consecutive paused repetitions at $87.5 \% 1 \mathrm{RM}$ to demonstrate

159 execution of a predicted three-repetition-maximum (3Rep) with consideration to

160 fatigue accumulated following the 1RM protocol (2). Participants finally

161 completed three sets of eight continuous and dynamic repetitions (8Rep) using

$16270 \% 1 \mathrm{RM}(2)$. Multiple repetition sets were also separated by a 5-minute rest 
166 Following the 7-14 day interval, participants returned to the laboratory and

interval and assistance racking and un-racking the barbell was provided at the participants' choosing.

$$
\text { *** Figure } 1 \text { about here }{ }^{* * *}
$$

completed the SS trial. Participants were provided with an introduction to the Slingshot, and, identical to trial 1, participants' anthropometric characteristics were taken and a re-familiarisation to the procedures was provided prior to commencing the warm-up. Several different size selections were provided for the 'Slingshot' device, and were fitted to each participant according to the manufacturer's instructions. The same warm-up protocol was followed, and an identical rack height and grip width was implemented. All warm-up prior to, and including normalisation repetitions were taken without the use of the 'Slingshot', and all repetitions performed following the $70 \%$ normalisation were completed using the 'Slingshot'. The 'Slingshot' was worn across the elbow joint as recommended by the product manufacturers, and to avoid disruption to EMG signals on the triceps and pec placements (See Figure 2). The achieved Raw 1RM weight was performed as one of the 'SS' $1 \mathrm{RM}$ attempts (Raw max/SS), participants then proceeded to add weight and follow the same protocol as the 'Raw' trial until a separate 'SS' 1RM was achieved. Participants' 3Rep and 8Rep weight were established using $87.5 \%$ and $70 \%$ of the achieved 'SS' 1RM respectively, and were performed in the same manner described for the 'Raw' trial. All attempts throughout both trials were performed to the nearest $1 \mathrm{~kg}$. 
sEMG amplitude was collected via skin surface electrodes (SilveRest, Vermed, VT) from the pectoralis major, anterior deltoid, and triceps brachii of the participants' dominant side during all repetitions using a BioPac MP100 (BioPac Systems Inc., CA). Reference signals were provided via skin surface electrodes placed on the clavicularis and patella. Prior to applying the electrodes, the skin surface was prepared for collection by shaving, slightly abrading using sandpaper, and wiped with alcohol swabs (PDI Healthcare, NJ), in line with SENIAM guidelines (17). A small amount of SignaGel electrode gel (Parker Laboratories, NJ) was used on the centre of each electrode to aid signal quality. Electrodes were applied to the skin surface $\sim 2 \mathrm{~cm}$ apart and secured to the skin surface with masking tape if necessary. Due to the nature and placement of the 'Slingshot', electrodes for the pectoralis major were placed using medial and central clavicularis placements as suggested by Krol et al, (19) (See Figure 1). Electrode placement for both the anterior deltoid and triceps brachii were in line with recommendation by Perotto and colleagues (24). As participants performed bench press repetitions, the EMG amplitude of the pectoralis major, anterior deltoid, and triceps brachii (RMS) was collected using the Acqknowledge software for Windows (BioPac Systems Inc., CA) and saved for offline analysis.

A Celesco PT5A-125-S47-UP-10K-M6 linear transducer (Celesco, CA), connected to a BioPac MP100 data capture unit (BioPac Systems Inc., CA), was secured to the top of the power rack to measure participants' average barbell velocity (m/s) and bar displacement $(\mathrm{cm})$ during all bench press attempts. During both 'Raw' and 'SS' trials, a Velcro strap attached to the transducer cable was secured in a consistent, slightly off centre placement on the barbell. The position of the 
transducer was adjusted appropriately for each participant so that the cable ran

211 vertically during bench press execution. As participants performed bench press

212 repetitions, the velocity and displacement of the barbell was recorded using the 213 Acqknowledge software for Windows (BioPac Systems Inc., CA) and saved for 214 offline analysis.

All data were analysed using the Acqknowledge software for Windows (BioPac Systems Inc., CA). Repetition phases were defined in accordance with $(10,33)$. sEMG signals for the pectoralis major, anterior deltoid, and triceps brachii were 218 digitised individually at a sampling rate of 2,000 Hz and recorded in Volts. sEMG signals were root mean square (RMS) processed based on previous recommendations for research investigating neuromuscular activation levels

221 (16).. Average RMS was calculated for a moving window 100ms time period across the entire waveform for each activity. sEMG signals were then normalised against corresponding repetitions extracted from the set of 5 reps performed to a metronome at 70\%1RM (7) as part of the participants' warm-up.

Analysis of the transducer data was performed by highlighting only the concentric phase of the repetitions. Phases were defined in accordance with Van den Tillaar et al, (33). For both maximal and sub-maximal repetitions, the start of the concentric phase was identified as the first point at which velocity reached $0 \mathrm{~m} / \mathrm{s}$, indicating a change of direction. For maximal attempts, the phases of the concentric portion of the bench press were defined as per Van Den Tillaar et al,

232 (33) (See Figure 3 for representative trace). This involved defining the beginning of the pre-stick period (phase 1) by identifying the point at which velocity was 0 
$\mathrm{m} / \mathrm{s}$ at the end of the eccentric phase, identifying the stick point and beginning of

235 the stick period (phase 2) as the point of peak velocity during the concentric 236 phase, and identifying the post-stick period (phase 3) as the point at which 237 acceleration again crossed $0 \mathrm{~m} / \mathrm{s}^{2}$. The lift ended when velocity reached $0 \mathrm{~m} / \mathrm{s}$ at 238 the end of the concentric phase. Each phase and/or repetition was analysed 239 individually for total time (s), average bar speed (m/s), and the stick point was 240 identified (m) comparative to the total displacement.

\section{Statistical Analysis}

242 All statistical analyses were carried out in GraphPad, Prism (GraphPad Software, 243 CA). Where 2 groups were compared, a 2 tailed $t$-test was performed. Where 244 more than two groups were compared, a 1 way ANOVA was utilized with a 245 Tukey's HSD test. Where multiple comparisons were made across groups, a 2 246 way ANOVA was performed with a Bonforoni's multiple comparisons test. 247 Normality of data was tested using D'Agostinio-Pearson omnibus normality test. 248 Where data were not normally distributed then the non-parametric two tailed t249 tests were performed. When multiple comparisons were made on non-normal 250 data then a Friedman test was utilized with a Dunn's multiple comparisons test. 251 All data were reported as mean \pm SEM and significance was set as a $p$ value of $p$ $252 \leq 0.05$. Correlations were determined via a simple linear regression. 
All participants displayed an increase in absolute bench press performance 260 whilst using the "Slingshot" from $139.7 \pm 4.34 \mathrm{~kg}$ 'Raw' to $160.4 \pm 4.43 \mathrm{~kg}$ "SS" for an average increase of $\sim 20 \mathrm{Kg}$ (Figure $4 \mathrm{~A}$ ). The absolute gain from the 'Slingshot' was not related to the amount lifted (Figure 4B) but instead was highly correlated to the individuals bodyweight $\left(\mathrm{R}^{2}=0.334\right)$, indicating that despite all participants wearing an appropriately sized device, the larger participants were able to gain more from the 'Slingshot' (Figure 4C). The 'Raw' 1RM corresponded 266 closely to the calculated 'SS' 3Rep (Figure 4D). These data were plotted and correlated revealing that there was a highly significant correlation between participant's 'Raw' 1RM and 'SS' 3Rep (R²=0.9538) (Figure 4E).

During maximal 1RM bench press attempts, 'Raw' normalised triceps RMS 271 (169.64 $\pm 15.26 \%)$ was significantly higher than both 'Raw max/SS' and 'SS conditions (87.28 $\pm 5.84 \% \& 115.84 \pm 10.64 \%$ ) (Figure $5 \mathrm{~A})$. Normalised RMS

273 for the pectoralis was significantly lower in the 'Raw max/SS' condition 274 compared to the 'SS' condition (90.83 $\pm 6.97 \%$ vs. $117.8 \pm 11.27 \%)$ during $1 \mathrm{RM}$ 275 attempts (Figure 5A). Normalised RMS for all muscles (grouped) was 276 significantly reduced during 1RM performance for 'Raw max/SS' (95.58 \pm 5.47 $277 \%$ ) than during the 'Raw' condition (138.82 $\pm 9.42 \%$ ) (Figure 5A). Normalised 278 triceps RMS was also observed to be significantly higher during the 'Raw' 279 condition $(126.02 \pm 9.19 \%)$ than the 'SS condition (83.12 $\pm 9.97 \%)$ during a set 
of 3 repetitions (Figure 5B), and during both set 1 (108.15 $\pm 6.25 \%$ vs. $75.96 \pm$ $7.36 \%)$ and set $3(115.35 \pm 7.48 \%$ vs. $84.37 \pm 8.45 \%)$ of the multiple sets of 8 repetitions (Figure 5C).

Average barbell velocity was significantly greater across the whole concentric phase by $\sim 3$ fold for the 'Raw max/SS' condition $(0.29 \pm 0.02 \mathrm{~m} / \mathrm{s})$ when compared to the 'Raw' \& 'SS' conditions $(0.11 \pm 0.01 \mathrm{~m} / \mathrm{s} \& 0.10 \pm 0.01 \mathrm{~m} / \mathrm{s})$ higher in the 'SS' condition compared to the 'Raw' condition $(0.31 \pm 0.02 \mathrm{~m} / \mathrm{s}$ vs. $0.27 \pm 0.02 \mathrm{~m} / \mathrm{s}$ ), as was the average velocity of phase 1 (Figure $6 \mathrm{~A}$ ). There is a trend for phase 3 to have a lower velocity in the 'SS' condition compared to 'Raw'. There is a high degree of variability between the 'SS' and 'Raw' conditions when plotting these data as individual responses, whilst during phase 1 there is a consistent increase in phase 1 velocity ( 9 subjects increase) when wearing the 'Slingshot' (Figure 6B).

The displacement data demonstrates that there was no effect of wearing the 'Slingshot' on total displacement indicating that hand position was replicated accurately between trials and that the 'Slingshot' did not affect the range of 298 motion (Figure 6C). However, the 'Slingshot' significantly altered the displacement at which the stick point occurred (Figure 6C). The effect was small,

$300 \sim 1 \mathrm{~cm}$ higher in the concentric phase, but very consistent with 12 out of 15 301 subjects demonstrating an upward shift in the start of the stick point (Figure 6C and 6D). The sticking period tended to occupy a greater proportion of the concentric phase whilst wearing the 'Slingshot' however this did not reach 
significance. Whilst not significant, when the individual data were plotted as $\%$ of the total displacement there was a similar trend but a high degree of intersubject variability, with 7 subjects demonstrating an increase and 8 subjects demonstrated a decrease in the stick period length whilst wearing the 'Slingshot' (Figure 6D).

No significant differences were found for average barbell velocity $(\mathrm{m} / \mathrm{s})$ during reps 1 and 2 of the 3Rep, however average barbell velocity was significantly faster for the 'SS' condition than the 'Raw' condition $(0.21 \pm 0.02 \mathrm{~m} / \mathrm{s}$ vs. $0.18 \pm 0.02 \mathrm{~m} / \mathrm{s}$ ) during rep 3 of the 3Rep (Figure 6E). Similarly, the change in barbell velocity (\%) between reps 1 and 3 of the 3 Rep were significantly lower in the 'SS' than the 'Raw' condition (-15.72 $\pm 5.36 \%$ vs. $-25.57 \pm 9.4 \%$ ) (Figure 6F). Average barbell velocity was significantly faster during the 'SS' than the 'Raw' condition for both set 1 rep $8(0.35 \pm 0.04 \mathrm{~m} / \mathrm{s}$ ys. $0.30 \pm 0.04 \mathrm{~m} / \mathrm{s})$ and set 3 rep 8 $(0.34 \pm 0.07 \mathrm{~m} / \mathrm{s}$ vs. $0.26 \pm 0.03 \mathrm{~m} / \mathrm{s})$ during the multiple sets of 8 repetitions (Figure 6G). Similarly, the change in barbell velocity (m/s) between reps 1 and 8 of the multiple sets of 8 were significantly lower in the 'SS' than the 'Raw' condition (-19.38 $\pm 4.4 \%$ vs. $-31.18 \pm 4.82 \%$ ) (Figure $6 \mathrm{H})$.

To determine the mechanism behind the improved 1RM performance whilst wearing the 'Slingshot' we split the analysis of the prime mover RMS over the 3 phases of the bench press; pre-stick period [1], stick period [2], and post-stick period [3]. These data revealed that there was no effect of the 'Slingshot' on pectoralis (Figure 7B) or deltoid (Figure 7C) RMS activation. However, the triceps RMS was significantly lower during the stick [2] and post-stick [3] periods (Figure 7A). 


\section{Discussion:}

330 This is the first study to assess the impact of the "Slingshot" bench press training 331 aid on bench press kinematics and neuromuscular activity in trained 332 powerlifters across a range of intensities. The 'Slingshot' was found to be an 333 effective elastic assistance device for enhancing 1RM bench press performance 334 in all participants, on average producing a fixed absolute increase of $\sim 20 \mathrm{~kg}$. This elastic assistance allowed the 'Raw' 1RM to be lifted with an average velocity

$336 \sim 3 \mathrm{x}$ faster than the 'Raw' 1RM performed unassisted. The increased velocity 337 whilst wearing the 'Slingshot' occurred in spite of significantly reduced RMS in 338 the triceps brachii. Furthermore, when intensity was matched (same relative $339 \% 1 \mathrm{RM}$ with or without the 'Slingshot') the RMS of the triceps was reduced at all 340 intensities. During both of the multiple repetition conditions (3Rep/8Rep), the 341 last rep was performed with a higher velocity than the corresponding 'Raw' condition. This preservation of barbell velocity throughout a set may be 343 indicative of reduced fatigue (26) suggesting that despite matching for relative 344 intensity, the 'Slingshot' may effectively reduce fatigue.

345 Components of the velocity data from our study are somewhat reminiscent of the 346 velocity data obtained from performing the bench press with chain weight (6). At 347 the beginning of the concentric phase, the assistance from the device will likely 348 be greatest, and like with chains, the force required to move the bar off the chest 349 will be lower with the weight experienced increasing into lockout. Therefore, 350 increased velocity is an attractive theory for the mechanism of how the 351 'Slingshot' may work. Despite the greater load, the average barbell velocity of the 
maximal lift was the same between conditions, with peak barbell velocity significantly faster during a maximal attempt with the 'Slingshot'. Assessing barbell velocity during the different pressing phases revealed that during the pre-stick period [1], barbell velocity was significantly faster whilst wearing the 'Slingshot'. The velocity data yields some insight into the mechanism by which the 'Slingshot' may allow an individual to complete a full lift with significantly more weight than their 'Raw' $1 \mathrm{RM}$. We initially theorized that as the concentric phase progressed, the activation of the prime movers would increase to compensate for the reducing assistance supplied by the elastic device. To our surprise the RMS of the pectoralis and the deltoids was the same between the 'Raw' and 'SS' conditions, whilst the RMS of the triceps was significantly reduced in the 'SS' trial. These data indicate that despite $\sim 20 \mathrm{~kg}$ extra on the bar, the triceps are having to activate to a lesser extent to complete the lift, even during the post-stick [3] phase of the lift where the assistance from the device would be assumed to be minimal. We hypothesize that the ability to complete the repetition with a significantly greater load during the 'SS' trial is driven by both the increased peak velocity, and increased velocity throughout the pre-stick [1] period imparting more momentum to the barbell. However, it is unlikely that increased velocity is the mechanism responsible for every individual, as several individuals display a reduced velocity during the pre-stick period [1]. As a result, we must explore additional theories as to how the 'Slingshot' allows individuals to lift significantly higher loads, despite similar, and in some cases reduced, prime mover sEMG. 
One possibility is that the 'Slingshot' may alter the mechanics of the bench press by pulling the elbows into a more mechanically advantageous position. Van den Tillaar et al, (33), suggest that the stick period during the bench press is partly due to the arm position transitioning into a less mechanically advantageous position. It is possible that due to the nature and placement of the 'Slingshot', that it may be maintaining the elbows in a position which may allow for a stronger press. Another possibility for how the 'Slingshot' alters the bench press performance is that the 'Slingshot' shifts the displacement at which the stick period begins. Whilst the shift in where the stick period begins was small, $\sim 1 \mathrm{~cm}$ higher during 'SS' vs 'Raw', we cannot rule out the possibility that this small shift may have had a significant effect on the ability to complete the lift.

The study by Ye et al, (35), similar to ours, found a fixed increase in 1RM performance from wearing the 'Slingshot'. They observed an increase in absolute $1 \mathrm{RM}$ performance from $114.6 \mathrm{~kg}$ to $132.1 \mathrm{~kg}$ and a fixed mean increase of $17.6 \mathrm{~kg}$ whilst wearing the 'Slingshot'. Our findings however, showed that the 'Slingshot' improved mean $1 \mathrm{RM}$ bench press performance from $139.7 \mathrm{~kg}$ to $160.4 \mathrm{~kg}$ with a mean fixed increase of $20.67 \mathrm{~kg}$. Although largely similar, the slight differences in findings between our study and those of Ye et al, (35) are possibly due to the training status and technical competency of the different sample groups. As in our study, they also found no correlation between the 1RM and the gain from wearing the 'Slingshot'. These data indicate that the weight lifted whilst wearing the 'Slingshot' is not related to the amount of weight on the bar. We find however, that the mass of the individual is significantly correlated to the gain from wearing the 'Slingshot'. The range in gain from wearing the 'Slingshot' in 
our study was $15-27 \mathrm{~kg}$ with the greatest gain achieved by the largest (by body mass) individual in the study $(124.1 \mathrm{~kg})$. We theorize that this effect may be driven by chest girth i.e. greater chest girth creating a greater stretch producing more elastic assistance. Future studies should correlate more comprehensive anthropometric measures to the gain from wearing the 'Slingshot' thus allowing for an individual to estimate the gain they will get from the device by making a simple anthropometric measure.

\section{Practical applications:}

Aside from suggesting how the 'Slingshot' works, our data also suggest some potential uses for the device in training. Some researchers have theorized that the benefit of elastic training is that it allows for similar forces to be produced but at faster velocities (20). The 'Slingshot' could be used as a speed training device, as our data clearly demonstrate that velocity is substantially improved whilst wearing the 'Slingshot'. Therefore, it may have some utility in velocity training for sports such as the shot-put. However, the sEMG data shows that the triceps are very likely de-loaded at all intensities with the 'Slingshot'. These findings combined with the velocity data from the multiple repetition sets suggest that the 'Slingshot' likely reduces fatigue and could also be used as a deloading tool. One advantage of the 'Slingshot' over other de-loading or speed training tools, such as using bands and chains, is its ease of use. Furthermore, unlike other commonly used de-loading tools, the 'Slingshot' allows for a full range of motion to be performed as demonstrated by total barbell displacement during both 'Raw' and 'SS' trials. It should be noted that if the 'Slingshot' was employed for a bulk of training, that the potential de-loading of the triceps would 
In summary, the acute increases observed in bench press performance resultant

speed training and de-loading the bench press exercise during a variety of

the bench press.

\section{References:}

1. Anderson CE, Sforzo GA, and Sigg JA. The effects of combining elastic and free weight resistance on strength and power in athletes. The Journal of Strength \& Conditioning Research 22: 567-574, 2008.

2. Arazi $\mathrm{H}$ and Asadi A. The relationship between the selected percentages of one repetition maximum and the number of repetitions in trained and untrained males. Facta universitatis-series: Physical Education and Sport 9: 25-33, 2011.

3. Argus CK, Gill ND, Keogh JW, Blazevich AJ, and Hopkins WG. Kinetic and training comparisons between assisted, resisted, and free countermovement jumps. $J$ Strength Cond Res 25: 2219-2227, 2011.

4. Atkinson G and Reilly T. Circadian variation in sports performance. Sports medicine 21: 292-312, 1996.

5. Baechle TR, and Earle, R.W. Essentials of Strength Training and Conditioning. Champaign, Ill: Human Kinetics, 2000.

6. Baker DG and Newton RU. Effect of kinetically altering a repetition via the use of chain resistance on velocity during the bench press. The Journal of Strength \& Conditioning Research 23: 1941-1946, 2009.

7. Balshaw TG and Hunter AM. Evaluation of electromyography normalisation methods for the back squat. J Electromyogr Kinesiol 22: 308-319, 2012.

8. Bartolini JA, Brown LE, Coburn JW, Judelson DA, Spiering BA, Aguirre NW, Carney KR, and Harris KB. Optimal elastic cord assistance for sprinting in collegiate women soccer players. J Strength Cond Res 25: 1263-1270, 2011.

9. Bellar DM, Muller MD, Barkley JE, Kim CH, Ida K, Ryan EJ, Bliss MV, and Glickman EL. The effects of combined elastic- and free-weight tension vs. free-weight tension on one-repetition maximum strength in the bench press.J Strength Cond Res 25: 459-463, 2011.

10. Elliott BC, Wilson GJ, and Kerr GK. A biomechanical analysis of the sticking region in the bench press. Med Sci Sports Exerc 21: 450-462, 1989.

11. Fahey T. Predictors of performance in elite discus throwers. Biology of Sport 19: 103-108, 2002.

12. Garrido N, Marinho DA, Barbosa TM, Costa AM, Silva AJ, Pérez Turpin JA, and Marques MC. Relationships between dry land strength, power variables and short sprint performance in young competitive swimmers. 2010.

13. Ghigiarelli JJ, Nagle EF, Gross FL, Robertson RJ, Irrgang JJ, and Myslinski T. The effects of a 7-week heavy elastic band and weight chain program on upper-body 
strength and upper-body power in a sample of division 1-AA football players. The Journal of Strength \& Conditioning Research 23: 756-764, 2009.

14. Giorgio P, Samozino P, and Morin J-B. Multigrip flexible device: Electromyographical analysis and comparison with the bench press exercise. The Journal of Strength \& Conditioning Research 23: 652-659, 2009.

15. Glass SC and Armstrong T. Electromyographical Activity of the Pectoralis Muscle During Incline and Decline Bench Presses. The Journal of Strength \& Conditioning Research 11: 163-167, 1997.

16. Hägg G, Melin B, and Kadefors R. Applications in ergonomics. Electromyography: Physiology, Engineering, and Non-invasive Applications: 343-363, 2004.

17. Hermens HJ, Freriks B, Disselhorst-Klug C, and Rau G. Development of recommendations for SEMG sensors and sensor placement procedures. Journal of electromyography and Kinesiology 10: 361-374, 2000.

18. Jones MT. Effect of compensatory acceleration training in combination with accommodating resistance on upper body strength in collegiate athletes. Open access journal of sports medicine 5: 183, 2014.

19. Krol H, Sobota G, and Nawrat A. Effect of electrode position on EMG recording in pectoralis major. Journal of Human Kinetics 17: 105, 2007.

20. Leontijevic B, Pazin N, Kukolj M, Ugarkovic D, and Jaric S. Selective effects of weight and inertia on maximum lifting. Int J Sports Med 34: 232-238, 2013.

21. McCaw ST and Friday JJ. A Comparison of Muscle Activity Between a Free Weight and Machine Bench Press. The Journal of Strength \& Conditioning Research 8: 259-264, 1994.

22. McKean MR and Burkett BJ. The influence of upper-body strength on flat-water sprint kayak performance in elite athletes. International Journal of Sports Physiology \& Performance 9, 2014.

23. Ogasawara R, Thiebaud RS, Loenneke JP, Loftin M, and Abe T. Time course for arm and chest muscle thickness changes following bench press training. Interventional Medicine and Applied Science 4: 217-220, 2012.

24. Perotto A and Delagi EF. Anatomical guide for the electromyographer: the limbs and trunk. Charles C Thomas Publisher, 2005.

25. Sadri I, Jourkesh M, Ostojić SM, Calleja-Gonzalez J, Ojagi A, and Neshati A. A Comparison of EMG Fluctuation of Deltoid and Pectoralis Major Muscles in Bench Press. Sport Science: 30, 2011.

26. Sanchez-Medina L and González-Badillo JJ. Velocity loss as an indicator of neuromuscular fatigue during resistance training. Med Sci Sports Exerc 43: 17251734, 2011.

27. Soria-Gila MA, Chirosa IJ, Bautista IJ, Baena S, and Chirosa LJ. Effects of Variable Resistance Training on Maximal Strength: A Meta-Analysis. The Journal of Strength \& Conditioning Research 29: 3260-3270, 2015.

28. Swinton P, Keogh J, and Lake J. Practical Applications of Biomechanical Principles in Resistance Training: The use of Bands and Chains. Journal of Fitness Research 3, 2014.

29. Swinton PA, Lloyd R, Agouris I, and Stewart A. Contemporary training practices in elite British powerlifters: Survey results from an international competition. The Journal of Strength \& Conditioning Research 23: 380-384, 2009.

30. Tan B. Manipulating Resistance Training Program Variables to Optimize Maximum Strength in Men: A Review. The Journal of Strength \& Conditioning Research 13: 289-304, 1999.

31. Tran TT, Brown LE, Coburn JW, Lynn SK, Dabbs NC, Schick MK, Schick EE, Khamoui AV, Uribe BP, and Noffal GJ. Effects of different elastic cord assistance levels on vertical jump. The Journal of Strength \& Conditioning Research 25: 3472-3478, 2011. 
32. van den Tillaar R and Ettema G. A comparison of successful and unsuccessful attempts in maximal bench pressing. Med Sci Sports Exerc 41: 2056-2063, 2009.

33. Van Den Tillaar R and Ettema G. The "sticking period" in a maximum bench press. Journal of sports sciences 28: 529-535, 2010.

34. Wilson J and Kritz M. Practical Guidelines and Considerations for the Use of Elastic Bands in Strength and Conditioning. Strength \& Conditioning Journal 36: 1-9, 2014.

35. Ye X, Beck, T.W., Stock, M.S., Fahs, C.A., Kim, D., Loenneke, J.P., Thiebaud, R.S., Defreitas, J.M., Rossow, L.M., Bemben, D.A., and Bemben, M.G. Acute Effects of Wearing an Elastic, Supportive Device on Bench Press Performance in Young, Resistance-Trained Males. Gazzetta Medica Italiana 173: 91-102, 2014.

\section{Acknowledgements}

This study was conducted without any financial support and we have no conflicts of interest. The results of this study do not constitute endorsement of the product by the authors or the NSCA. We gratefully acknowledge expert technical assistance for Mr Chris Grigson. Finally we are indebted to the volunteers who gave theirt ime to this study.

\section{Figure Legends:}

Figure1. 'Slingshot' placement during a representative repetition. Subjects were instructed to perform the 'Slingshot' repetitions to the same IPF standards as in the previous 'Raw' trials.

Figure2. Electrode placement and positioning of the 'Slingshot.' Participants were instructed to wear the 'Slingshot' with the crease centred at the elbow. Electrodes were placed as described in the methods to ensure that the 'Slingshot' did not disrupt the electrodes during bench pressing.

Figure3. Defining the phases of maximal bench press attempts. Figure 3 illustrates a representative trace of acceleration, velocity and displacement during a 1RM attempt. The beginning of the pre-stick period (phase 1) was identified as the point at which velocity was $0 \mathrm{~m} / \mathrm{s}$ at the end of the eccentric phase. The stick point, and the beginning of the stick period (phase 2) was identified as the point of peak velocity during the concentric phase. The post-stick period (phase 3) began when acceleration again crossed $0 \mathrm{~m} / \mathrm{s}^{2}$. The post-stick period ended when velocity reached $0 \mathrm{~m} / \mathrm{s}$ at the end of the concentric phase.

Figure4. The 'Slingshot' increases the bench press 1 repetition maximum in a manner correlated to body mass. Fifteen trained power-lifters underwent rep max testing on two occasions separated by 7-14 days. The 'Raw' repetition maximum (1RM) was determined without any assistance and the SS repetition maximum was determined whilst wearing the 'Slingshot.' After the 1RM testing, 3 repetitions (3Rep) were 
performed at $87.5 \%$ of the achieved $1 \mathrm{RM}$ followed by 3 sets of 8 (8Rep) at $70 \%$ of the achieved 1RM. The mean weight lifted in each of these conditions is plotted in (A) with the individual 1RM data plotted on the inset graph in (A). The absolute gain from wearing the 'Slingshot' (the difference between Raw and SS trials) was plotted against the Raw 1RM achieved (B) and against the body mass of the individuals (C). The individual data for the Raw 1RM and the weight lifted on the 3Rep SS trial were plotted (D) with a linear regression of these variables plotted in (E). *indicates significantly different from corresponding 'Raw' condition (as assessed by paired t-test between respective 'Raw' and 'SS' conditions). Significance was determined as $\mathrm{p} \leq 0.05$.

Figure5. The 'Slingshot' reduces sEMG amplitude of the triceps brachii at all intensities. The 'Raw max/SS' was performed during the warm up of the SS trial day and consisted of performing the previous session's raw 1RM whilst wearing the 'Slingshot'. Surface EMG (sEMG) amplitudes were recorded during all sets and reps as described in the methods. All data presented are root mean squared (RMS) processed and normalised to the $70 \%$ normalisation set. A) sEMG amplitudes recorded during repetition maximum testing, B) SEMG amplitudes recorded during the set of 3 repetitions at $87.5 \%$ and C) sEMG amplitudes recorded during the 3 sets of 8 repetitions at $70 \%$. $\Phi$ indicates 'Raw' is significantly different from both 'Raw max/SS' and 'SS' (as assessed by multiple comparisions). $\alpha$ indicates significantly different from 'SS' (as assessed by multiple comparisons). $\S$ indicates significantly different from 'Raw' (as assessed by multiple comparisons). * indicates significantly different from 'Raw' (assessed by paired t-test). Significance was determined as $\mathrm{p} \leq 0.05$. Tricep - triceps brachii, pec - pectoralis clavicularis, delt - anterior deltoid, grouped - sEMG grouped for all 3 muscles assessed.

Figure6. The 'Slingshot' improves peak barbell velocity on maximal efforts and maintains mean barbell velocity in multiple repetition sets. Barbell velocity was tracked during all movements using a vertical transducer. The phases of the bench press were determined by assessing the acceleration curves with the stick period defined as the period between negative and positive barbell acceleration. A) Barbell velocity during rep max testing. B) Individual changes in barbell velocity between 'Raw' and 'SS' trials assessed by phases of the maximal effort. C) Barbell displacement during maximal efforts with the displacement at which the stick point occurs and also the displacement over which the stick period lasts also plotted. D) The \% of the total displacement at which the stick period begins (stick point) and the \% displacement over which the stick period lasts plotted as individual responses from the 'Raw' to the 'SS' trials. E) Average barbell velocity for each repetition of the set of 3 reps at $87.5 \%$. F) The $\%$ decrement in barbell velocity from repetition 1 to repetition 3 on the 'Raw' and 'SS' trials. G) Average barbell velocity for the first and last rep of the first and last set of the 3 sets of 8 repetitions at 70\%. H) The \% decrement in barbell velocity from repetition 1 to repetition 8 on the third set of the 'Raw' and 'SS' trials. $\Phi$ indicates 'Raw' is significantly different from both 'Raw max/SS' and 'SS.' $\alpha$ indicates significantly different from 'SS' (assessed by multiple comparisons). $\varepsilon$ indicates significant difference between two bars (assessed by multiple comparisons). * indicates significantly different from 'Raw' (assessed by paired t-test). Significance was determined as $\mathrm{p} \leq 0.05$. 
Figure7. The 'Slingshot' reduces sEMG amplitude of the triceps brachii during a maximal effort. Surface EMG (sEMG) amplitudes were recorded during repetition maximum testing as described in the methods. All data presented are root mean squared (RMS) processed and normalised to the $70 \%$ normalisation set. A) sEMG amplitudes of the triceps brachii, B) SEMG amplitudes of the pectoralis clavicularus and C) SEMG amplitudes of the anterior deltoids. $\alpha$ indicates significantly different from 'SS' (as assessed by multiple comparisons $\mathrm{p} \leq 0.05$ ). 


\begin{tabular}{|l|l|l|l|l|l|l|}
\hline & $\begin{array}{l}\text { Age } \\
(\mathrm{yrs})\end{array}$ & $\begin{array}{l}\text { Mass } \\
(\mathrm{kg})\end{array}$ & $\begin{array}{l}\text { Height } \\
(\mathrm{cm})\end{array}$ & $\begin{array}{l}\text { Bench } \\
\text { Press } \\
1 \mathrm{RM} \\
(\mathrm{kg})\end{array}$ & $\begin{array}{l}\text { Training } \\
\text { Age } \\
(\mathrm{yrs})\end{array}$ & $\begin{array}{l}\text { Training } \\
\text { Sessions/ } \\
\text { wk (days) }\end{array}$ \\
\hline $\mathrm{N}=15$ & $\begin{array}{l}27.05 \\
\pm 5.94\end{array}$ & $\begin{array}{l}94.15 \\
\pm \\
13.43\end{array}$ & $\begin{array}{l}177.38 \\
\pm 4.33\end{array}$ & $\begin{array}{l}139.73 \\
\pm 16.79\end{array}$ & $\begin{array}{l}5.93 \pm \\
5.67\end{array}$ & $4.2 \pm 0.53$ \\
\hline
\end{tabular}




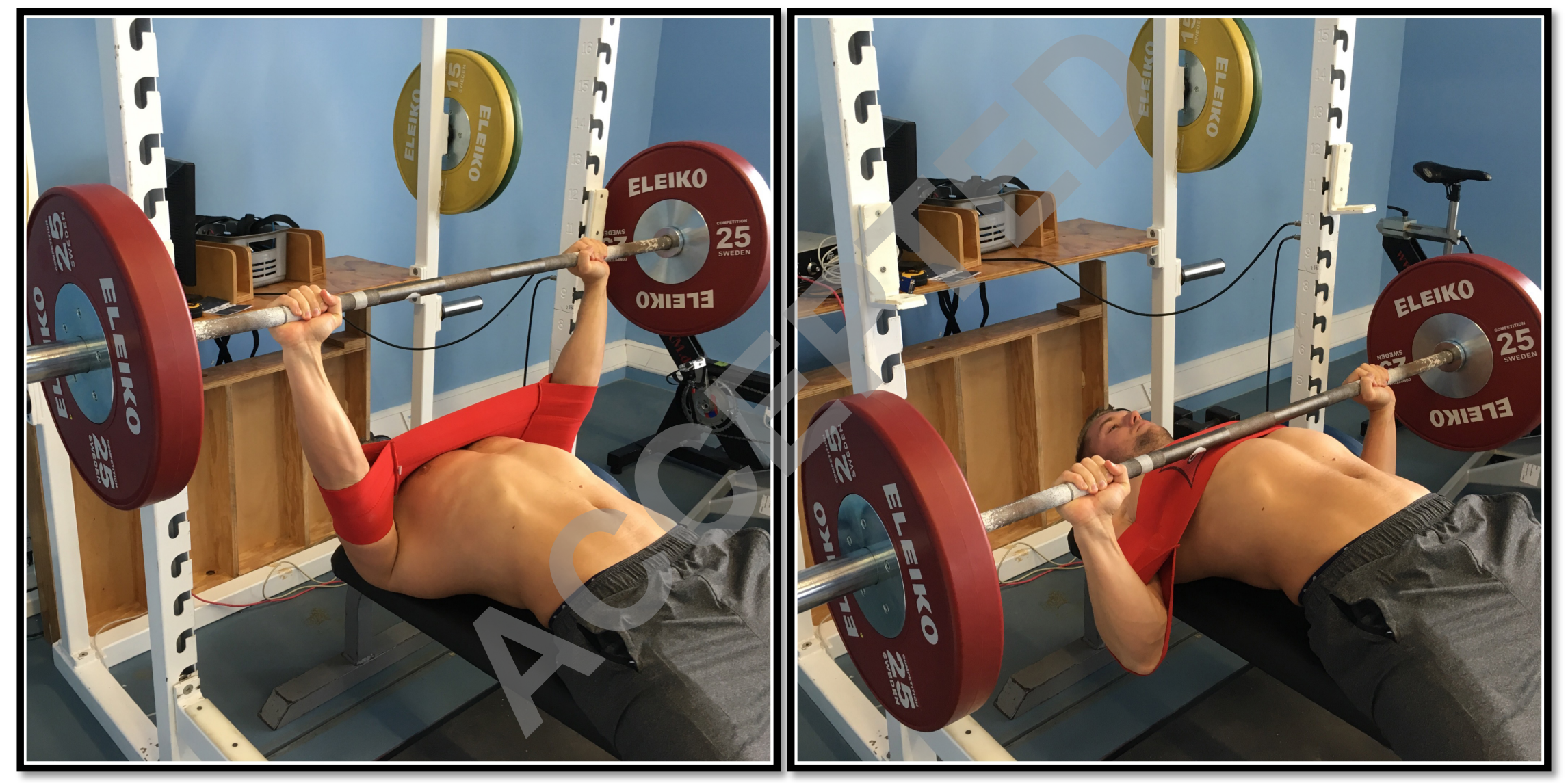




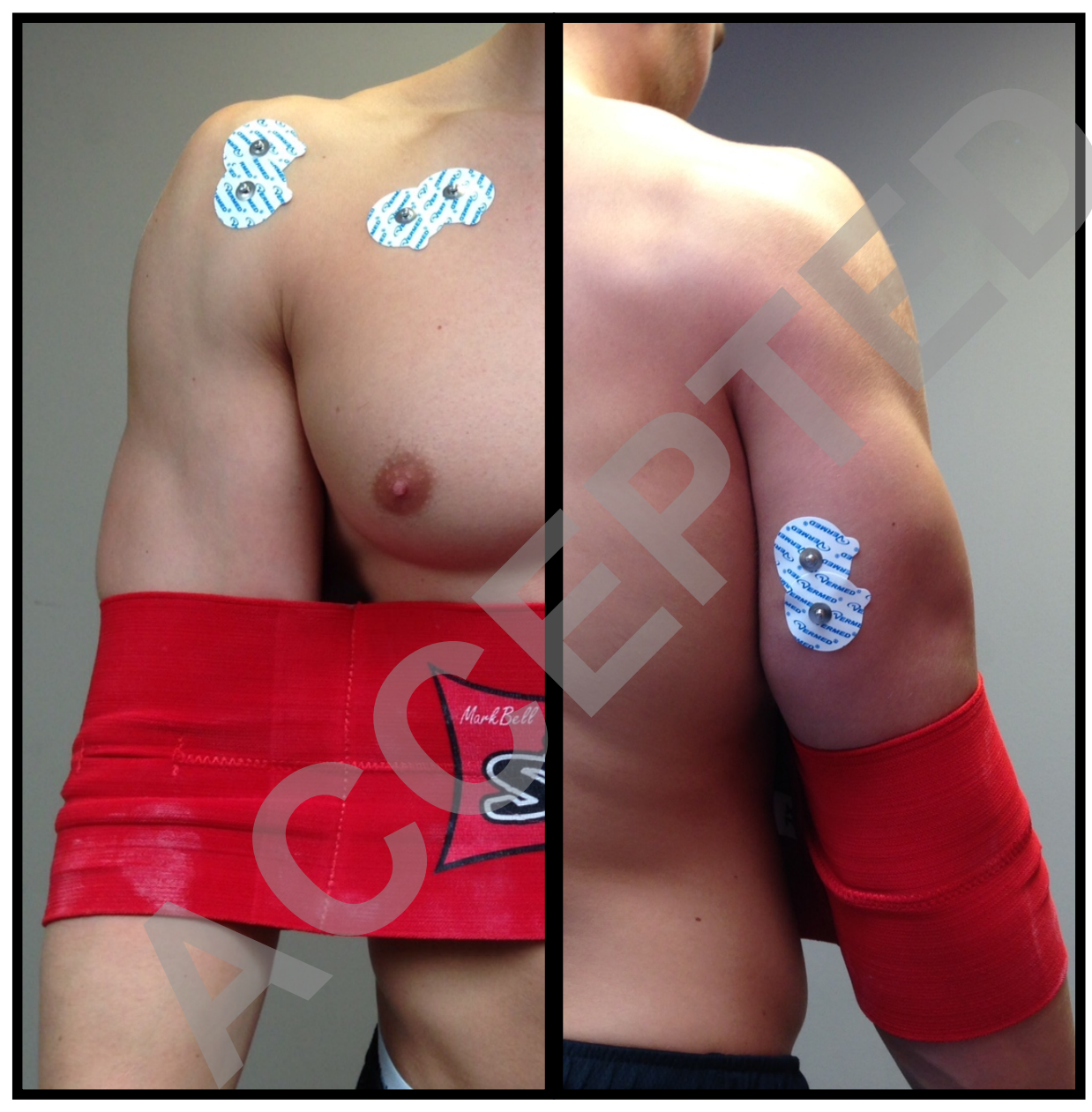




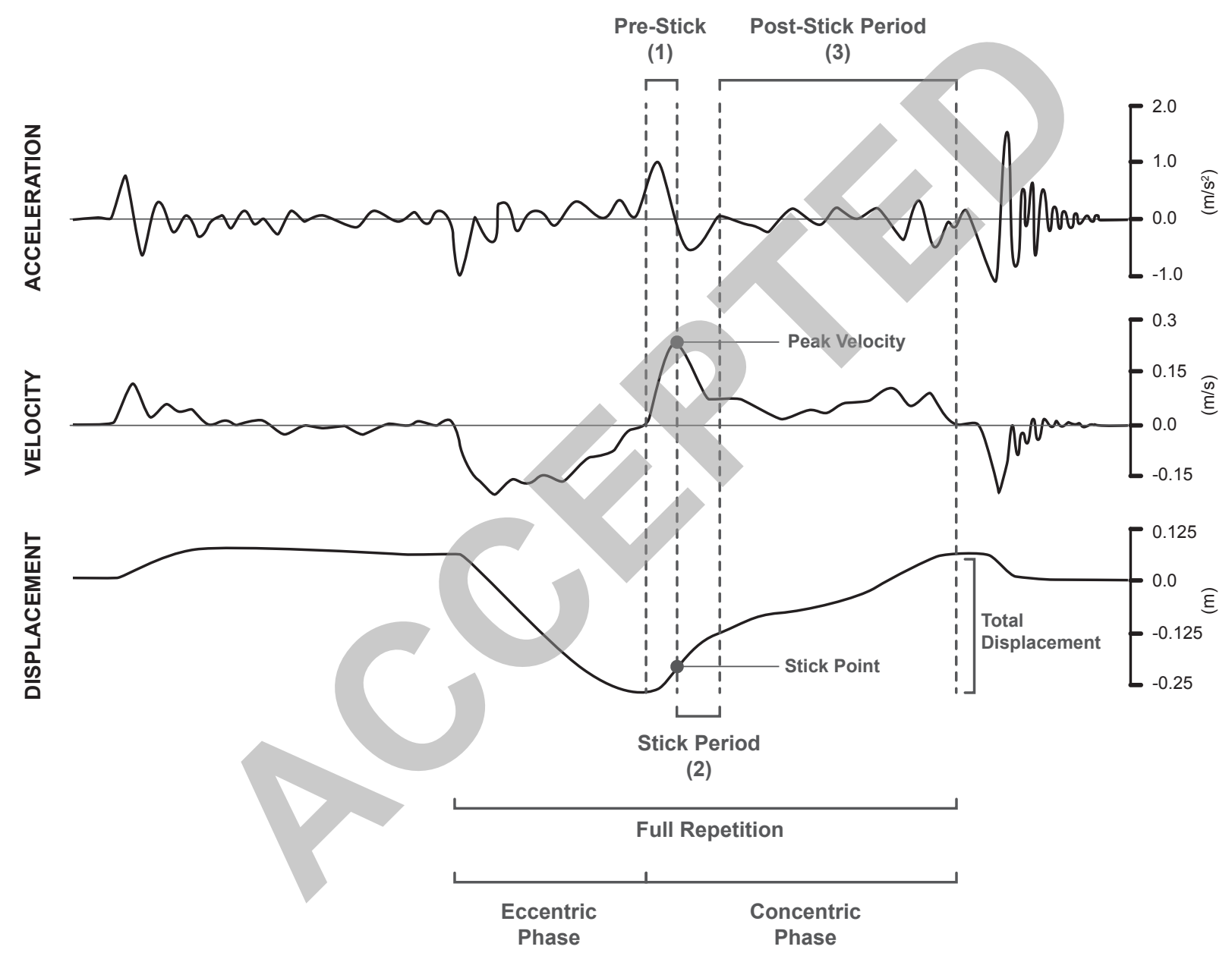



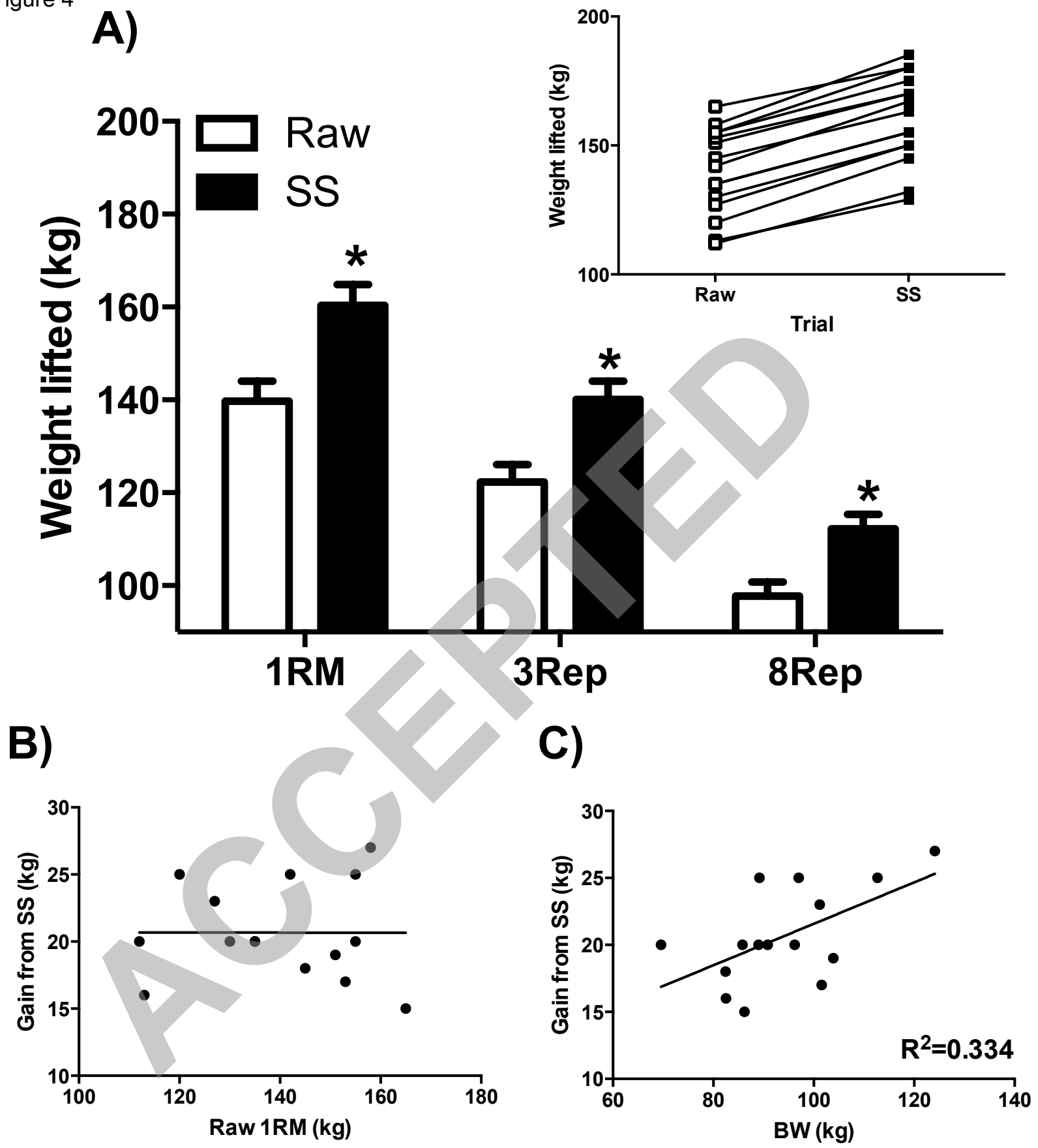

D)

E)

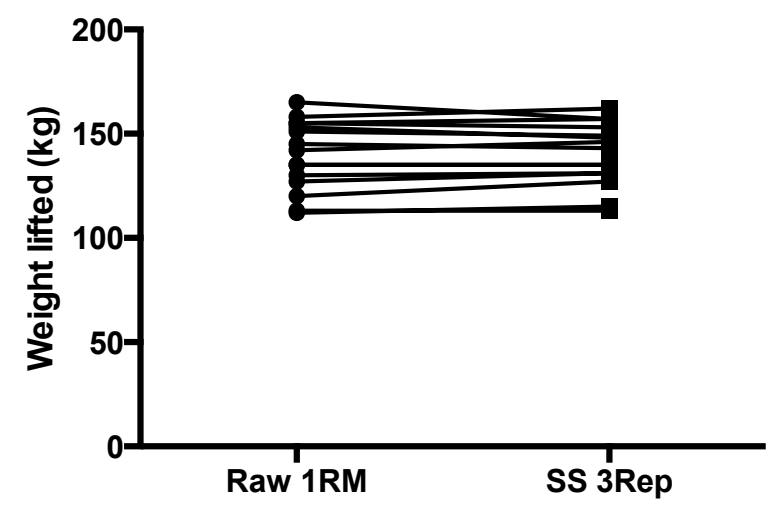

Attempt
Raw 1RM (kg)
Copyright (C) 2017 National Strength and Conditioning Association

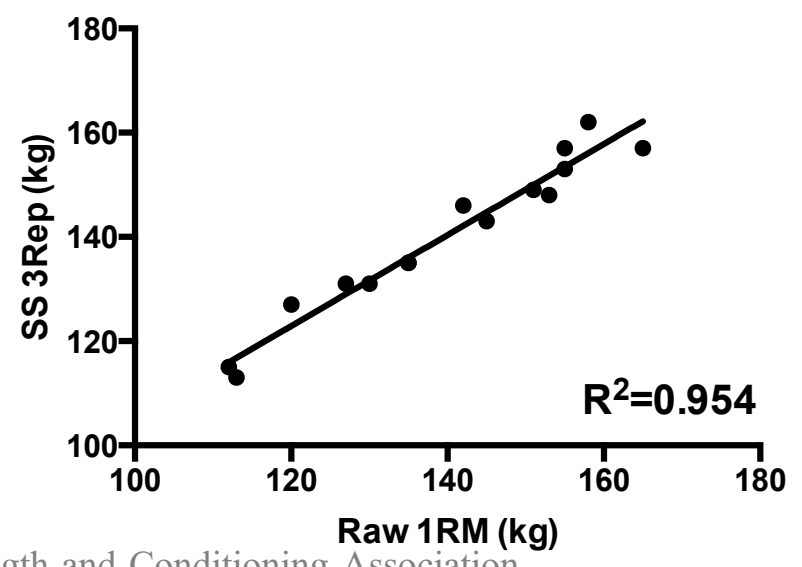




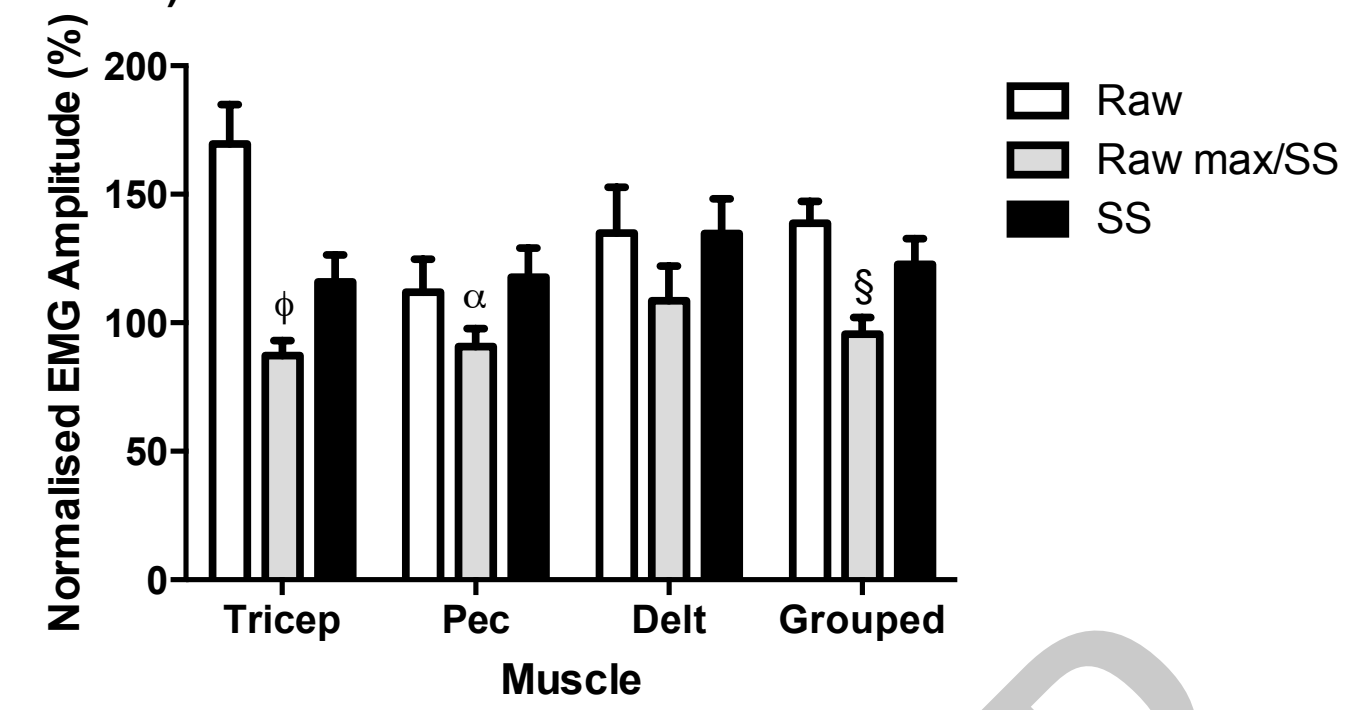

B)
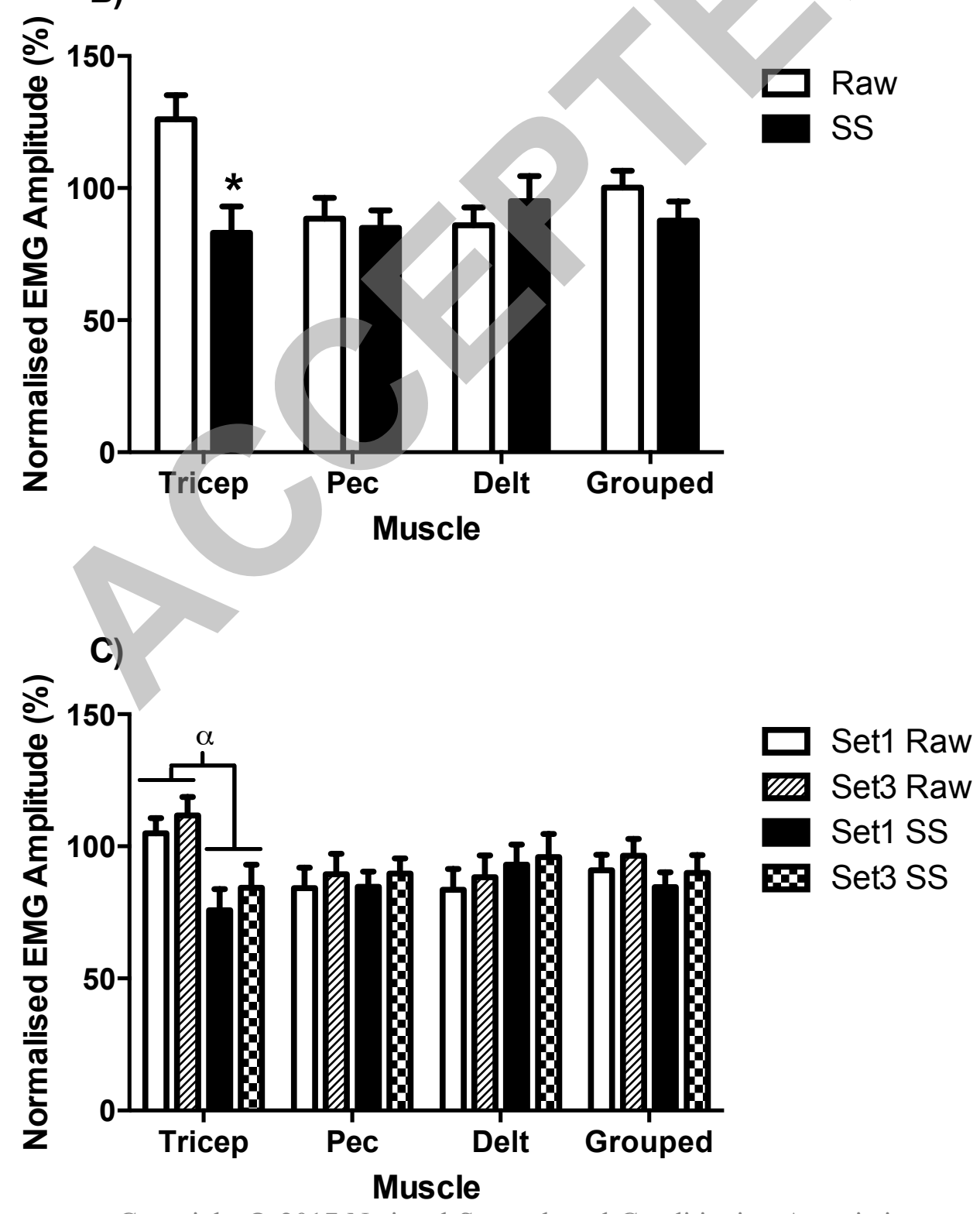

Copyright (C) 2017 National Strength and Conditioning Association 
A)

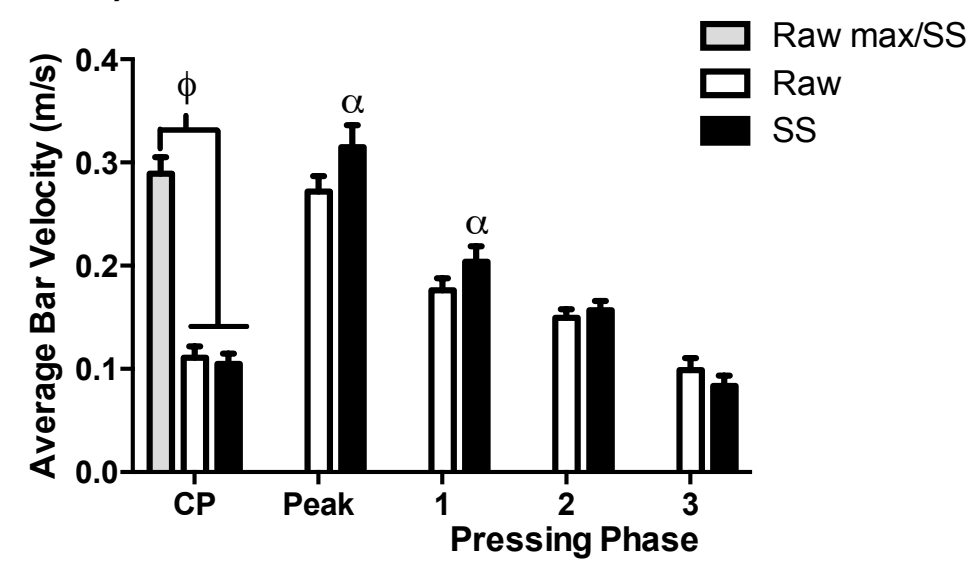

C)

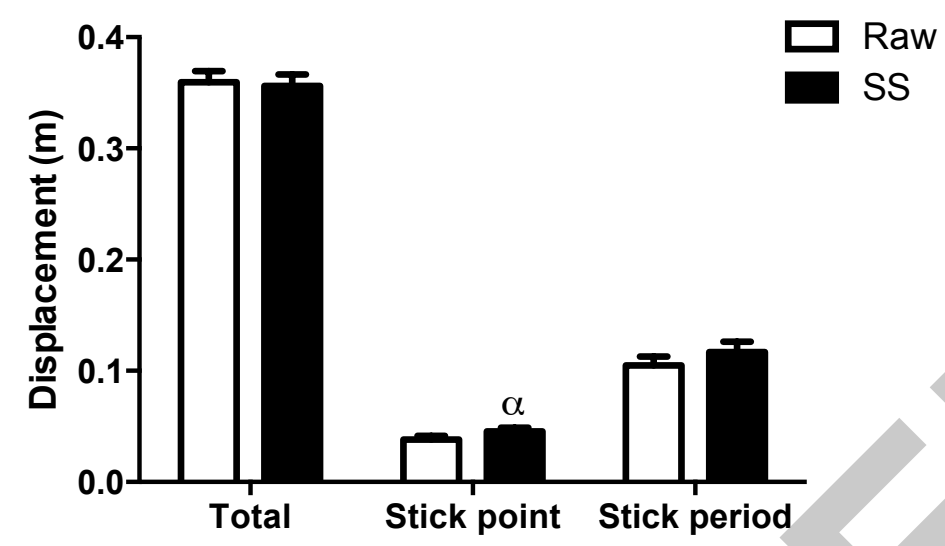

E)

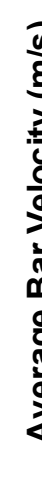

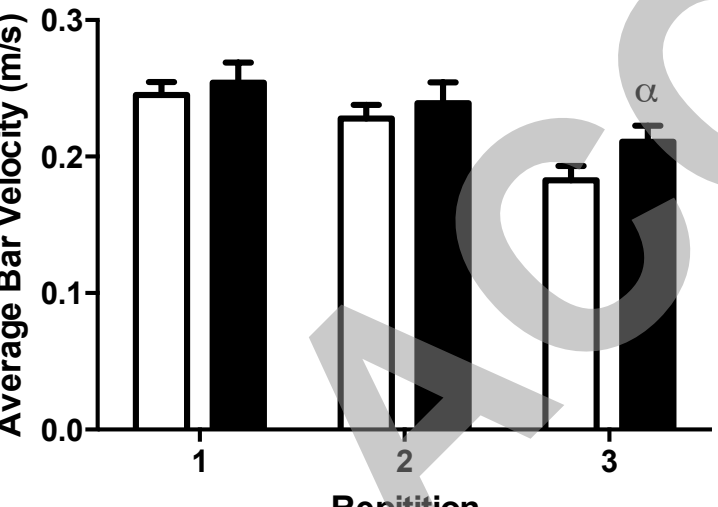

Repitition

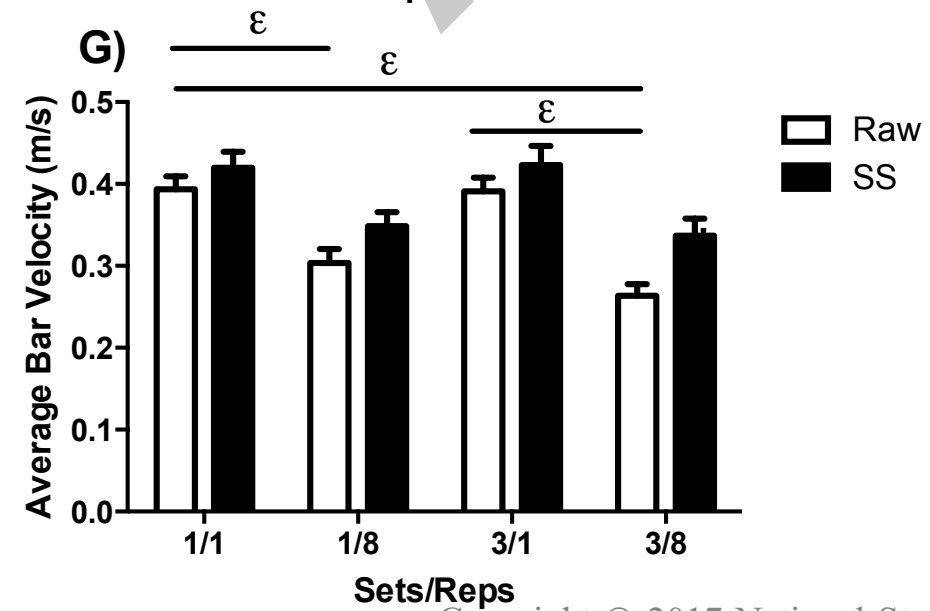

B)

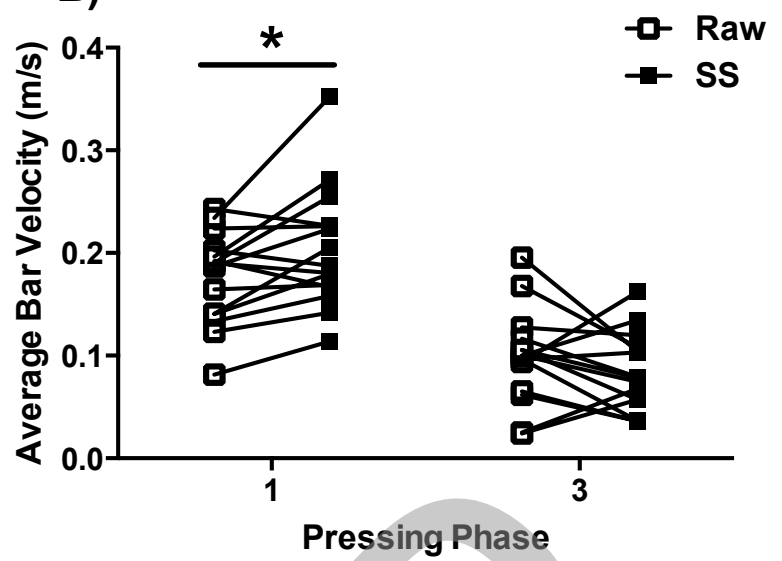

D)
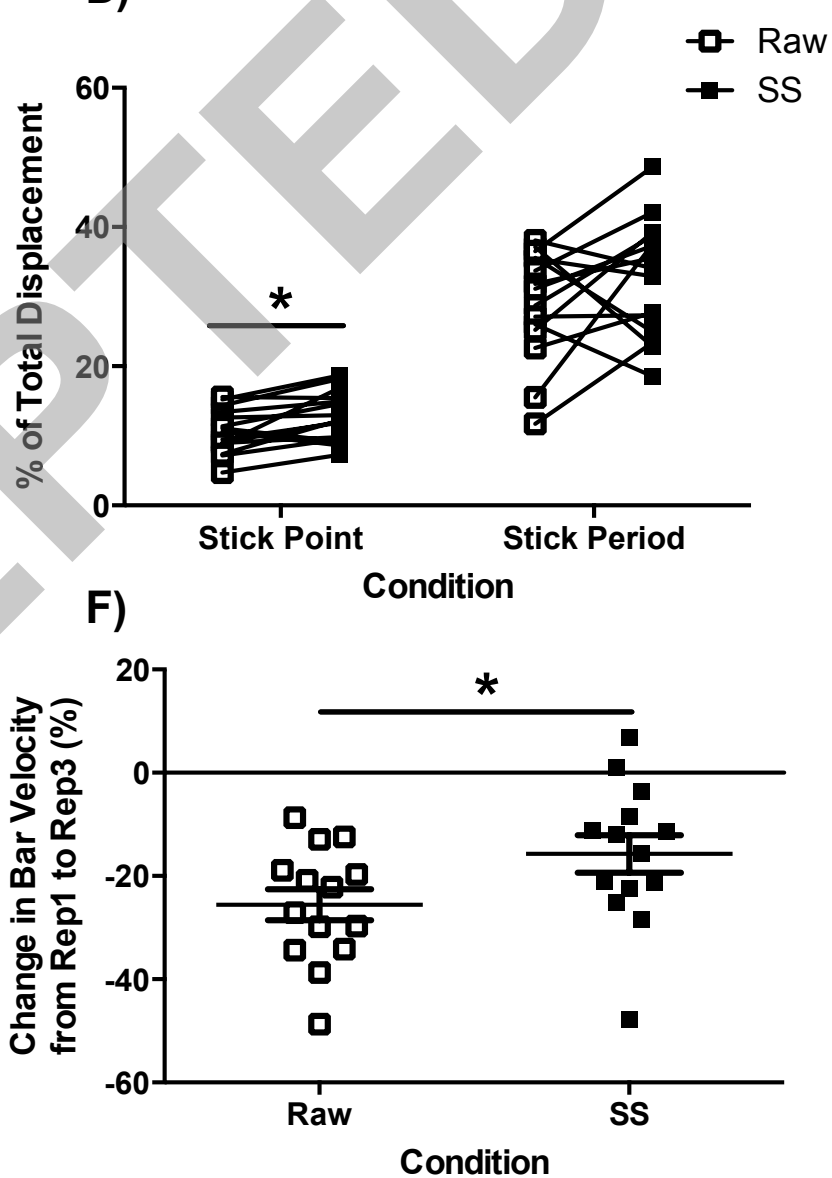

H)

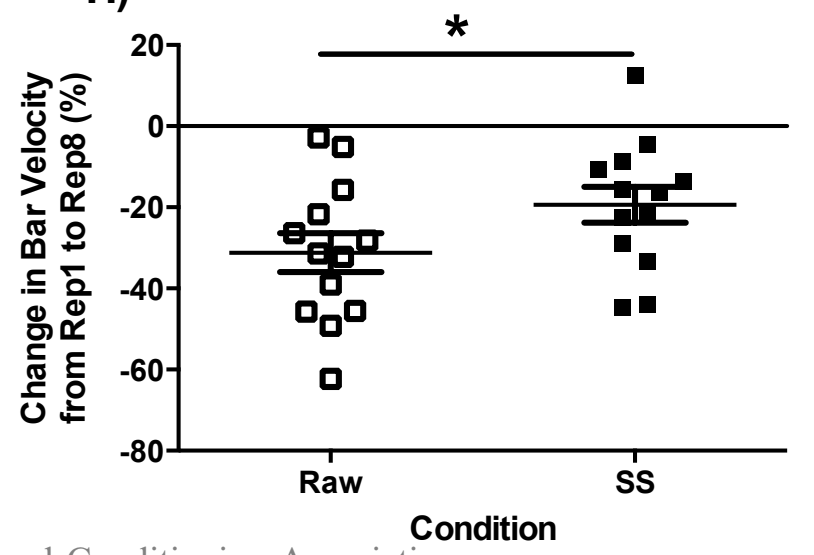


Aigore 7

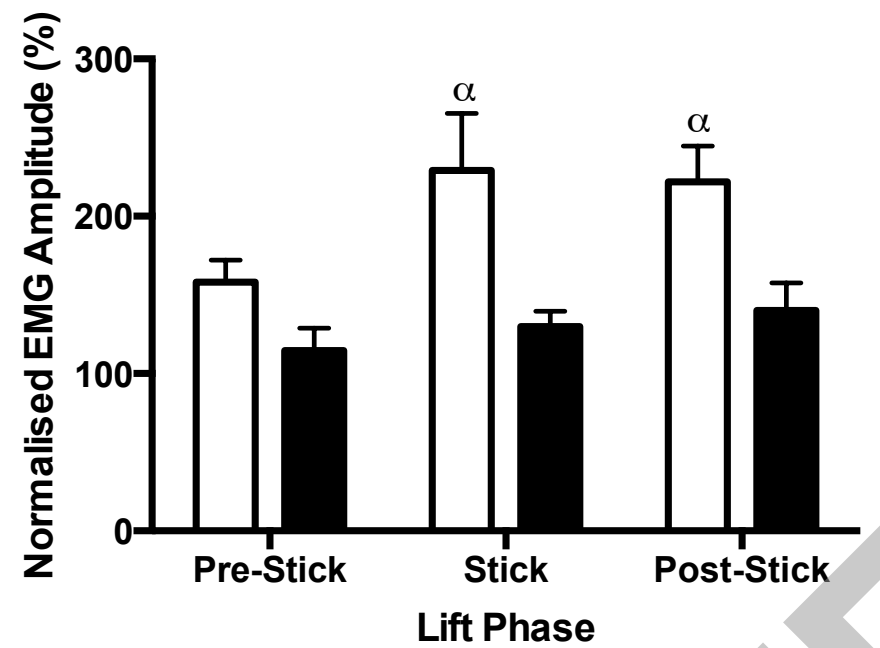

B)

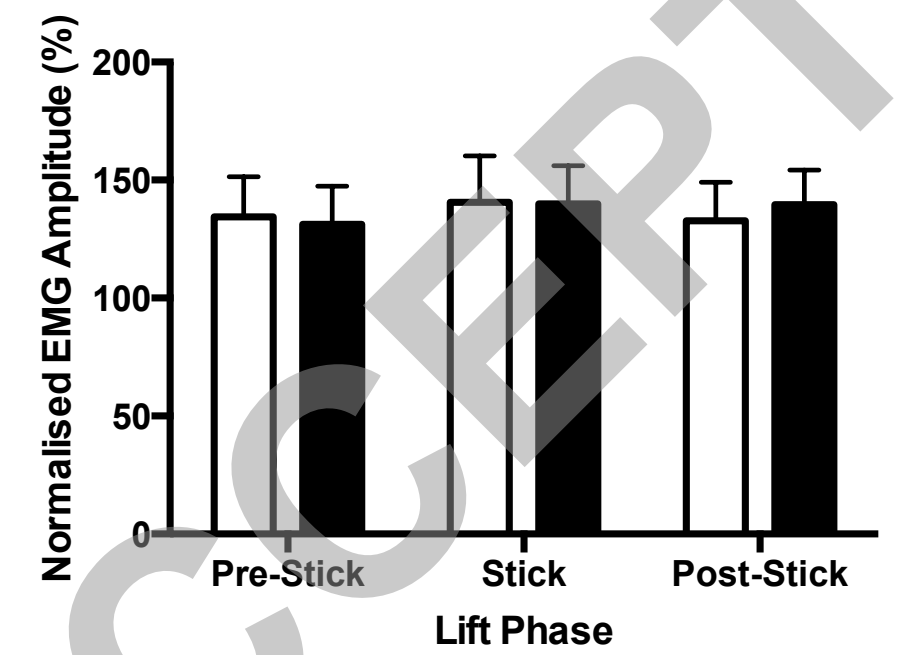

C)

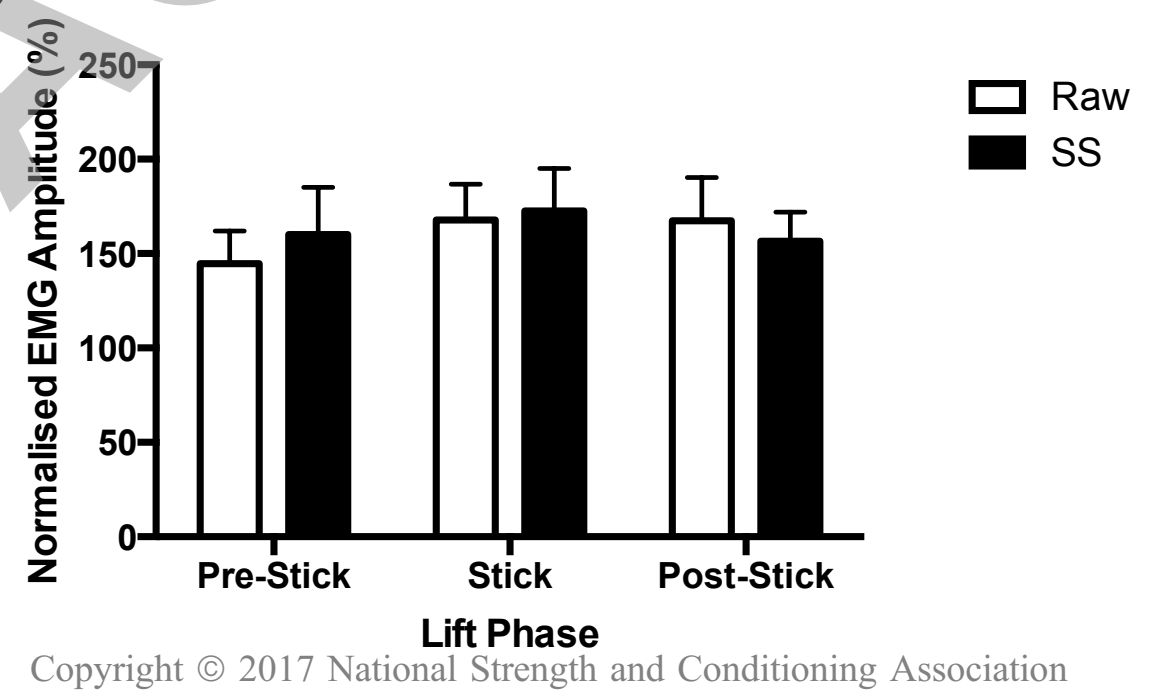

